\title{
TANGENT LINES AND PLANES IN TOPOLOGICAL SPACES*
}

BY

\section{CHARLES C. TORRANCE}

The object of this paper is to extend to topological spaces some of the concepts and theorems of direct infinitesimal geometry. To attain this object we define certain point sets in a topological space which will play the role of lines, planes, and hyperplanes in Euclidean space. By means of these sets we define the terms tangent line, tangent plane, etc. We arrive at our principal results in Theorems 4 and $4 \mathrm{a}$.

Let $S$ be a point space with a system of neighborhoods satisfying

Axıом 1. There exists a sequence $\left\{G_{n}\right\}, n=1,2, \cdots$, of collections of neighborhoods in $S$ such that (1) for each $n, G_{n}$ covers $S$; (2) for each $n, G_{n+1}$ is a subcollection of $G_{n}$; (3) if $R$ is any neighborhood in $S$, and if $X$ and $Y$ are points of $R$, distinct or not, there exists an integer $m$ such that, if $g$ is any neighborhood of the collection $G_{m}$ containing $X$, then $\bar{g}$ is a subset of $(R-Y)+X$; and (4) if $H$ and $K$ are mutually exclusive closed point sets in $S$ and if $H$ is compact, there exists an integer $m$ such that no neighborhood of the collection $G_{m}$ contains both a point of $H$ and $a$ point of $K$.

This axiom consists of the first four parts of R. L. Moore's Axiom 1' $\dagger$ and is sufficient $\ddagger$ to establish the theorems to be quoted below. In particular, point sequences and their sequential limits have the various properties§ required in

Defrnition 1. If $\left\{A_{n}\right\}$ is a sequence of point sets in the space $S$, then the sequential limiting set of $\left\{A_{n}\right\}$ is the set of all points $P$ such that there exists a sequence $\left\{P_{n}\right\}$ of points with sequential limit $P$, where $P_{n}$ is in $A_{n}$ for each $n$; the limiting set of $\left\{A_{n}\right\}$ is the set of all points $P$ such that there exist a subsequence $\left\{n_{i}\right\}$ of the sequence $\{n\}$ and a sequence $\left\{P_{n_{i}}\right\}$ of points with sequential limit $P$, where $P_{n_{i}}$ is in $A_{n_{i}}$ for each $n_{i}$.

* Presented to the Society, in part, September 12, 1935, under a slightly different title; received by the editors June 9,1936 . The main portion of this paper was presented to the International Congress of Mathematicians at Oslo, July 17, 1936.

$\dagger$ Moore, Foundations of Point Set Theory, Colloquium Publications of the American Mathematical Society, vol. 13, New York, 1932, p. 324. Hereafter, we shall designate this reference by the letter $\mathrm{M}$.

$\ddagger M$, p. 82.

$\S \mathrm{M}$, Chapter I, Theorems 2,3 , and 11 . 
REMARK 1 . If, in the preceding definition, all the sets $A_{n}$ have a common subset $M$, then the limiting set $A$ and the sequential limiting set $A^{\prime}$ of $\left\{A_{n}\right\}$ each contain $\bar{M}$; if $A_{n}=M$ for each $n$, then $A=A^{\prime}=\bar{M}$.

Definition 2. If $G$ and $H$ are two collections of point sets in the space $S$, then $G$ is called $H$-continuous provided that (1) if $g$ is any element of $G$ and $\left\{g_{n}\right\}$ is any sequence of elements of $G$ such that, $\gamma$ being the sequential limiting set of $\left\{g_{n}\right\}$, the part of $\gamma$ contained in $g$ is not contained in any element of $H$, then, $\left\{B_{n}\right\}$ being an arbitrary sequence of points with $B_{n}$ in $g_{n}$ for each $n$, each subsequence of $\left\{B_{n}\right\}$ contains a subsequence having a sequential limit in $g$; and (2) in the preceding notation, each point $C$ of $g$ is the sequential limit of some sequence $\left\{C_{n}\right\}$ of points, where $C_{n}$ is in $g_{n}$ for each $n$.

REMARK 2 . It is apparent that the preceding definition is not essentially altered if merely the sequence $\left\{B_{n}\right\}$ itself is required to contain a subsequence having a sequential limit in $g$. Nor is the preceding definition essentially altered if each point of $g$ is required to be merely the sequential limit of a sequence $\left\{C_{n_{i}}\right\}$, where $\left\{n_{i}\right\}$ is some subsequence of the sequence $\{n\}$.

Lemma 1. If $G$ is an $H$-continuous collection in $S$, and if $M$ is a point set in $S$ which is contained in no element of $H$, then there exists at most one element $g$ of $G$ which contains $M$.

Suppose there exist two distinct elements $g$ and $g^{\prime}$ of $G$ which contain $M$. Let $P$ be a point of one of these elements, say $g^{\prime}$, which is not in the other. Let $\left\{g_{n}\right\}$ be the sequence $g^{\prime}, g^{\prime}, g^{\prime}, \ldots$. By Remark $1, M$ is a subset of the part of the sequential limiting set of $\left\{g_{n}\right\}$ contained in $g$. Hence $g$ and $\left\{g_{n}\right\}$ are in the relation described in Definition 2. If $\left\{B_{n}\right\}$ is taken to be the sequence $P, P, \cdots$, then $P$, contrary to hypothesis, is in $g$.

REMARK 3. If $G$ is an $H$-continuous collection in $S$, and if $g$ and $h$ are any two distinct elements of $G$, then it follows from Lemma 1 that some element of $H$ contains the common part of $g$ and $h$.

Lemma 2. If $G$ is an $H$-continuous collection in $S$, and if no element of $G$ is contained in any element of $H$, then the elements of $G$ are closed and compact.

Let $g$ be an element of $G$ and let $P$ be a limit point of $g$. There exists a sequence $\left\{P_{n}\right\}$ of points of $g$ such that $\lim P_{n}=P$. Let $\left\{g_{n}\right\}$ be the sequence $g, g, \cdots$. Then $g$ and $\left\{g_{n}\right\}$ are in the relation described in Definition 2 since $g$ is contained in no element of $H$. If $\left\{B_{n}\right\}$ is taken to be $\left\{P_{n}\right\}$, it follows that $P$ is in $g$. The proof of compactness is similar.

Notation. If $H$ is a collection of point sets, then $H^{*}$ denotes the point set sum of all the elements of $H$. 
DefInItion 3. If $G$ is a collection of point sets such that $G^{*}=S$, then a subcollection $R$ of $G$ is called an element neighborhood provided that (1) $R^{*}$ is open, and (2) if $g$ is an element of $G$ such that $g^{*} \subset R^{*}$, then $g$ is an element of $R$.

If $G$ is a collection of point sets such that $G^{*}=S$, then $G$ itself is an element space which may be topologized in the usual manner by means of the element neighborhoods just now defined. Thus it has meaning to speak of a limit element, of a closed set of elements, of an element continuum, etc.

Notation. If $G$ is a collection of point sets, and if $U, V, \cdots$ comprise a collection $H$ of point sets, then $r_{U V} \ldots$ denotes the collection of all those elements $g$ of $G$ such that $g$ contains at least one point of each element of $H$, and $R_{U V} \ldots$ denotes $\dagger$ the collection of all those elements $g$ of $G$ such that $g^{*} \subset r_{U V}^{*} \ldots$

If in Definition 2, $H$ is the collection of all those point sets which consist of a single point, then $G$ is called point continuous. Moreover, $g$ and $\left\{g_{n}\right\}$ are in the relation described in Definition 2 when, and only when, there exist two sequences $\left\{A_{n}\right\}$ and $\left\{A_{n}^{\prime}\right\}$ of points with distinct sequential limits in $g$, where $A_{n}$ and $A_{n}^{\prime}$ are in $g_{n}$ for each $n$.

Definitron 4 . The elements of a collection $G^{(1)}$ of continua in the space $S$ are called lines if (1) $G^{(1)}$ is point continuous; (2) if $P$ and $Q$ are distinct points in $S$, there exists at least one element of $G^{(1)}$ containing $P$ and $Q$; and (3) if $U$ and $V$ are mutually exclusive neighborhoods in $S$ containing the points $P$ and $Q$ respectively, there exist neighborhoods $U^{\prime}$ and $V^{\prime}$ of $P$ and $Q$ within $U$ and $V$ such that $r_{U^{\prime} V^{\prime}}=R_{U^{\prime} V^{\prime}}$, where $r_{U^{\prime} V^{\prime}}$ and $R_{U^{\prime} V^{\prime}}$ are defined relative to $G^{(1)}$.

In what follows, all the point sets considered lie in the space $S$; moreover, it will be supposed that $G^{(1)}$ is a given collection of lines in $S$ and that the word "line" always connotes "line of $G^{(1)}$." By Lemma 1, there exists a unique line containing any two distinct points $P$ and $Q$; this line will sometimes be denoted by $\overline{P Q}$.

REMARK 4. If $P$ is a point, there exists a neighborhood of $P$ which contains no line. To show this, let $Q$ be a point distinct from $P$. There exists $\ddagger$ a pair of mutually exclusive neighborhoods $U$ and $V$ of $P$ and $Q$. If each neighborhood $U^{\prime}$ of $P$ within $U$ contains a line, then Condition (3) of Definition 4 cannot be met since $U^{\prime} \cdot V=0$ and $U^{\prime} \subset R_{U^{\prime} V^{\prime}}^{*}$, where $V^{\prime}$ is any neighborhood of $Q$ within $V$. It follows from this remark that every line contains more than one point.

† We use the notation $R_{U V} \ldots$ because of Lemmas 3 and $3 \mathrm{a}$; in what follows, the collection $G$, relative to which $R_{U V} \ldots$ is defined, is always explicitly understood.

$\ddagger$ M. Chapter I, Theorem 23. 
ExAmple 1. In the Euclidean space $E_{4}$ of four dimensions, let $\lambda$ be the family of planes

$$
\left\{\begin{array}{l}
a x-b y+z=m \\
b x+a y+w=n
\end{array}\right.
$$

together with the planes

$$
\left\{\begin{array}{l}
x=\alpha \\
y=\beta
\end{array}\right.
$$

The elements of $\lambda$ are lines in the sense of Definition 4 when only a bounded convex portion of $E_{4}$ is considered, since two points determine an element of $\lambda$.

Lемма 3. If $U$ and $V$ are mutually exclusive open point sets, then the set of lines $R_{U V}$ is a line neighborhood.

The proof of this lemma is effected in two stages. Let $A$ be a point not in $V$ and let $C$ be the "cone" of lines containing $A$ and a point of $V$. Suppose that $C^{*}-A$ is not open. Then there exists a point $P$ of $C^{*}-A$ which is the sequential limit of a sequence $\left\{P_{n}\right\}$ of points in the complement of $C^{*}-A$. Let $Q$ be a point of the line $\overline{A P}$ in $V$. By Condition (2) of Definition $2, Q$ is the sequential limit of a sequence $\left\{C_{n}\right\}$ of points, where $C_{n}$ is in the line $\overline{A P}_{n}$ for each $n$. By Lemma 1 , the lines $\overline{A P}_{n}$ have no point in common with $C^{*}-A$. Hence no point $C_{n}$ is in $V$. It follows that $C^{*}-A$ is open.

Now let $P$ be a point of $R_{U V}^{*}$. There exists a line $l$ containing $P$, a point of $U$, and a point of $V$. Let $A$ be a point of $l$ distinct from $P$ and in either $U$ or $V$, say $U$. Let $C$ be the cone of lines containing $A$ and a point of $V$. Then $P$ is in the open point set $C^{*}-A$. But $C^{*}-A \subset R_{U V}^{*}$. Hence $R_{U_{V}}^{*}$ is open. That $R_{U V}$ is a line neighborhood now follows from the definition of the symbol $R_{U V}$.

Three point sets are called non-collinear when there exists no line containing a point of each of these sets. Three non-collinear point sets are evidently mutually exclusive.

If in Definition 2, $H$ is the collection $G^{(\mathbf{1})}$, then $G$ is called line continuous. Moreover, $g$ and $\left\{g_{n}\right\}$ are in the relation described in Definition 2 when, and only when, there exist three sequences $\left\{A_{n}\right\},\left\{A_{n}^{\prime}\right\}$, and $\left\{A_{n}^{\prime \prime}\right\}$ of points with non-collinear sequential limits in $g$, where $A_{n}, A_{n}^{\prime}$, and $A_{n}^{\prime \prime}$ are in $g_{n}$ for each $n$.

Definition 4a. The elements of a collection $G^{(2)}$ of point continua in $S$ are called planes if (1) $G^{(2)}$ is line continuous; (2) if $P, Q$, and $R$ are non-collinear points, there exists at least one element of $G^{(2)}$ containing $P, Q$, and $R ;(3)$ if $U, V$, and $W$ are non-collinear point neighborhoods containing the points $P, Q$, 
and $R$, respectively, there exist neighborhoods $U^{\prime}, V^{\prime}$, and $W^{\prime}$ of $P, Q$, and $R$ within $U, V$, and $W$ such that $r_{U^{\prime} V^{\prime} W^{\prime}}=R_{U^{\prime} V^{\prime} W^{\prime}}$, where $r_{U^{\prime} V^{\prime} W^{\prime}}$ and $R_{U^{\prime} V^{\prime} W^{\prime}}$ are defined relative to $G^{(2)}$; and (4) if $P$ and $Q$ are distinct points, and if $g$ is an element of $G^{(2)}$ containing $P$ and $Q$, then $g^{*} \supset \overline{P Q}^{*}$.

In what follows, it will be supposed that $G^{(2)}$ is a given collection of planes in $S$ and that the word "plane" always connotes "plane of $G^{(2)}$." By Lemma 1, there exists a unique plane containing any three non-collinear points $P, Q$, and $R$; this plane will sometimes be denoted by $\overline{P Q R}$.

REMARK 4a. If $l$ is a line, there exists a line neighborhood $R$ of $l$ such that $R^{*}$ contains no plane. To show this, let $P_{1}$ and $P_{2}$ be distinct points of $l$ and let $Q$ be a point not in $l$. There exists a pair of mutually exclusive open point sets $D$ and $D^{\prime}$ containing $l$ and $Q$. It will be shown in Lemma 4 below that there exist mutually exclusive point neighborhoods $U$ and $V$ of $P_{1}$ and $P_{2}$ such that $R_{U V}^{*} \subset D$. If $W$ is a neighborhood of $Q$ within $D^{\prime}$, then $U, V$, and $W$ are non-collinear. Suppose that, for each neighborhood $U^{\prime}$ of $P_{1}$ within $U$ and each neighborhood $V^{\prime}$ of $P_{2}$ within $V, R_{U^{\prime} V^{\prime}}^{*}$ contains a plane. Then Condition (3) of Definition 4a cannot be met since $R_{U^{\prime} V^{\prime}}^{*} \cdot W=0$ and, by Condition (4) of Definition 4a, $R_{U^{\prime} V^{\prime}}^{*} \subset R_{U^{\prime} V^{\prime} W^{\prime}}^{*}$, where $W^{\prime}$ is any neighborhood of $Q$ within $W$. It follows from this remark that every plane contains more than one line.

EXAMPLE 1a. In the Euclidean space $E_{4}$, let the word line have its ordinary significance, but let "line" denote a plane of Example 1. Since every line is contained in some "line," the set of all planes in a bounded convex portion of $E_{4}$ is both line continuous and "line" continuous. But this set of planes fails to meet Condition (4) of Definition 4a with respect to "lines."

Example 2. Let $A, B$, and $C$ be non-collinear points in the space $S$. Let the flat $\pi_{A B C}$ be defined as the point set sum of all lines $l$ which contain a "vertex" and a point of the "opposite side" of the "triangle" $A B C$. If it is postulated that a line is contained in a flat if the line contains two distinct points of the flat, then a flat is a point continuum and the set of all flats is line continuous. To show this, let $\pi_{A B C}$ be a flat and let $A^{\prime}, B^{\prime}$, and $C^{\prime}$ be noncollinear points of $\pi_{A B C}$. Then $\pi_{A^{\prime} B^{\prime} C^{\prime}}=\pi_{A B C}$. (If $P$ is a point of $\pi_{A^{\prime} B^{\prime} C^{\prime}}$, and if $A^{\prime}, B^{\prime}$, and $C^{\prime}$ are in $\pi_{A B C}$, then $P$ is in $\pi_{A B C}$. Hence $\pi_{A^{\prime} B^{\prime} C^{\prime}} \subset \pi_{A B C}$. Thus, if $C^{\prime}$ is in $\overline{B C}$, then $C$ is in $\overline{B C^{\prime}}$ and $\pi_{A B C}=\pi_{A B C^{\prime}}$. The same result follows when $C^{\prime}$ is in $\overline{A C}$. If $C^{\prime}$ is in $\pi_{A B C}$ but not in $\overline{A C}$ or $\overline{B C}$, then either $\overline{C^{\prime} A}$ meets $\overline{B C}$ in a point $D$, or $\overline{C^{\prime} B}$ meets $\overline{A C}$, or $\overline{C^{\prime} C}$ meets $\overline{A B}$. In the first case, $\pi_{A B C}=\pi_{A B D}$ $=\pi_{A B C^{\prime}}$, the other cases being similar. It follows that $\pi_{A B C}=\pi_{A B C^{\prime}}=\pi_{A B^{\prime} C^{\prime}}$, $=\pi_{A^{\prime} B^{\prime} C^{\prime}}$.) Let $\left\{\pi_{n}\right\}$ be a sequence of flats containing three sequences $\left\{A_{n}\right\}$, $\left\{A_{n}^{\prime}\right\}$, and $\left\{A_{n}^{\prime \prime}\right\}$ of points with non-collinear sequential limits $A, A^{\prime}$, and 
$A^{\prime \prime}$ in a flat $\pi$, where $A_{n}, A_{n}^{\prime}$, and $A_{n}^{\prime \prime}$ are in $\pi_{n}$ for each $n$. It may be assumed that, for each $n, A_{n}, A_{n}^{\prime}$, and $A_{n}^{\prime \prime}$ are non-collinear. Hence $\pi_{n}=\pi_{A_{n} A_{n}^{\prime} A_{n}^{\prime \prime}}$. Let $\left\{B_{n}\right\}$ be a sequence of points with $B_{n}$ in $\pi_{n}$ for each $n$. For infinitely many $n$, either $\overline{A_{n} B_{n}}$ or $\overline{A_{n}^{\prime} B_{n}}$ or $\overline{A_{n}^{\prime \prime} B_{n}}$ has a point $D_{n}$ in common with the respective line $\overline{A_{n}^{\prime} A_{n}^{\prime \prime}}$ or $\overline{A_{n} A_{n}^{\prime \prime}}$ or $\overline{A_{n} A_{n}^{\prime}}$; say it is $\overline{A_{n} B_{n}}$. Since lines are point continuous, some subsequence of $\left\{D_{n}\right\}$ has a sequential limit $D$ in $\overline{A^{\prime} A^{\prime \prime}}$. Hence some subsequence of $\left\{B_{n}\right\}$ has a sequential limit $B$ in $\overline{A D}$ and therefore in $\pi_{A A^{\prime} A^{\prime}}$. By Remark 2, Condition (1) of Definition 2 is met. The proof of Condition (2) is similar. Thus the set of all flats is line continuous; by Lemma 2, every flat is closed. Since every flat is connected, it is a continuum. It follows that if Condition (3) of Definition 4a is postulated for flats, then flats are planes.

Lemma 3a. If $U, V$, and $W$ are non-collinear open point sets, then the set of planes $R_{U V W}$ is a plane neighborhood.

The proof of this lemma is effected in three stages. Let $A$ and $B$ be points which are non-collinear with the open set $W$. An argument similar to that used in the proof of Lemma 3 shows that the "sheaf" $\sigma$ of all planes containing $\overline{A B}$ and a point of $W$ is such that $\sigma^{*}-\overline{A B}^{*}$ is open. Now let $A$ be a point and let $V$ and $W$ be open sets such that $A, V$, and $W$ are non-collinear. An argument similar to the second argument used in the proof of Lemma 3 shows that the "bundle" $\beta$ of all planes containing $A$, a point of $V$, and a point of $W$ is such that $\beta^{*}-A$ is open. A repetition of this argument, using this last result, leads to the lemma.

Lemma 4. If $l$ is a line, if $D$ is an open point set containing $l^{*}$, and if $P$ and $Q$ are distinct points of $l$, then there exists a pair of mutually exclusive point neighborhoods $U$ of $P$ and $V$ of $Q$ such that $R_{U V}^{*} \subset D$.

Suppose there does not exist such a pair of neighborhoods. Let $\left\{U_{n}\right\}$ and $\left\{V_{n}\right\}$ be sequences of point neighborhoods which close down on $\dagger P$ and $Q$. It may be assumed that, for each $n, U_{n}$ and $V_{n}$ are mutually exclusive. For each $n$, there exists a point $Z_{n}$ of $r_{U_{n} V_{n}}^{*}$ not in $D$. Hence there exists, for each $n$, a line of $r_{U_{n} V_{n}}$ containing $Z_{n}$, a point $X_{n}$ of $U_{n}$, and a point $Y_{n}$ of $V_{n}$. Since $\lim X_{n}=P$ and $\lim Y_{n}=Q$, it follows by Definition 2 that some subsequence of $\left\{Z_{n}\right\}$ has a sequential limit $Z$ in $l$. But $D$ is open and contains $l$. Hence the original supposition is inadmissible.

Lemma 4a. If $p$ is a plane, if $D$ is an open point set containing $p^{*}$, and if $P, Q$, and $R$ are non-collinear points of $p$, then there exist non-collinear point neighborhoods $U, V$, and $W$ of $P, Q$, and $R$ respectively such that $R_{U V W}^{*} \subset D$.

$\dagger$ That is, $\Pi U_{n}=P, U_{n} \supset \bar{U}_{n+1}$, and if $T$ is any point neighborhood of $P, T \supset \bar{U}_{n}$ for some $n$. 
It was pointed out in Remark 4 a that there exist non-collinear neighborhoods $U, V$, and $W$ of $P, Q$, and $R$. The rest of the proof is similar to the proof of Lemma 4.

Lemma 5. If $l$ and $l_{1}$ are distinct lines, there exists an open point set containing $l^{*}$ but not $l_{1}^{*}$. If $p$ and $p_{1}$ are distinct planes, there exists an open set containing $p^{*}$ but not $p_{1}^{*}$.

The lemma follows immediately from Remarks 4 and $4 a$, the fact that two lines have at most one common point and two planes have at most one common line, and the fact that lines and planes are closed.

Definitron 5. If $H$ is a collection of lines and if $P$ and $Q$ are distinct points, then $P$ and $Q$ are called $H$-related if, for arbitrary point neighborhoods $U$ of $P$ and $V$ of $Q$, there exists a line $l \neq \overline{P Q}$ of $H$ which contains $a$ point of $U$ and $a$ point of $V$. If $H$ is a collection of planes and if $P, Q$, and $R$ are non-collinear points, then $P, Q$, and $R$ are called $H$-related if, for arbitrary point neighborhoods $U, V$, and $W$ of $P, Q$, and $R$, there exists a plane $p \neq \overline{P Q R}$ of $H$ which contains points of $U, V$, and $W$.

LEMMA 6. In order that a line $l$ be a limit line of a collection $H$ of lines it is necessary and sufficient that $l$ contain at least one pair of $H$-related points.

Necessity. Suppose no two distinct points of $l$ are $H$-related. Let $P$ and $Q$ be any two distinct points of $l$. By hypothesis, there exist point neighborhoods $U$ of $P$ and $V$ of $Q$ such that no line of $H$, except possibly $\overline{P Q}$, contains a point of $U$ and a point of $V$. It may evidently be assumed that $U$ and $V$ are mutually exclusive. By Condition (3) of Definition 4, and Lemma 3, there exists a line neighborhood $r_{U^{\prime} V^{\prime}}$ of $l$ which contains no line of $H$ except possibly $\overline{P Q}$. The original supposition is thus inadmissible.

Sufficiency. Suppose that $P$ and $Q$ are $H$-related points of $l$. Let $R$ be an arbitrary line neighborhood of $l$. By Lemma 4 , there exists a pair of mutually exclusive point neighborhoods $U$ of $P$ and $V$ of $Q$ such that $R_{U V} \subset R$. Since $P$ and $Q$ are $H$-related, $R_{U V}$, and hence also $R$, contain a line of $H$ other than $\overline{P Q}$.

LEMMA 6a. In order that a plane $p$ be a limit plane of a collection $H$ of planes it is necessary and sufficient that $p$ contain at least one set of three non-collinear $H$-related points.

The proof is similar to the proof of Lemma 6.

LemMa 7. If $R$ is a line neighborhood, then $\bar{R}^{*} \subset \overline{R^{*}}$.

Suppose there exists a point $A$ of $\bar{R}^{*}$ which is not contained in $\overline{R^{*}}$. Let $l$ be a line of $\bar{R}$ containing $A$. Then $l$ is a limit line of $R$. By Lemma $6, l$ contains 
at least one pair of $R$-related points $P$ and $Q$. Let $\left\{U_{n}\right\}$ and $\left\{V_{n}\right\}$ be sequences of point neighborhoods which close down on $P$ and $Q$ respectively. For each $n$, there exists a line $l_{n}$ of $R$ containing a point $P_{n}$ of $U_{n}$ and a point $Q_{n}$ of $V_{n}$. Since $\lim P_{n}=P$ and $\lim Q_{n}=Q$, it follows that $l$ and the sequence $\left\{l_{n}\right\}$ are in the relation described in Definition 2; but it is apparent that Condition (2) of Definition 2 cannot be met with regard to $A$.

Lemma 7a. If $R$ is a plane neighborhood, then $\bar{R}^{*} \subset \overline{R^{*}}$.

The proof is similar to the proof of Lemma 7.

THÉOREM 1. There exists a sequence $\left\{G_{n}{ }^{\prime}\right\}$ of collections of line neighborhoods satisfying Conditions (1), (2), and (3) of Axiom 1 when $S$ is interpreted to be $G^{(1)}$ and "point" is interpreted to be "line."

For each integer $n$ and each line $l$ let $D_{n l}$ be the sum of all the neighborhoods of $G_{n}$ which contain at least one point of $l$, and let $G_{n l}^{\prime}$ be the collection of all line neighborhoods $R$ such that $R^{*} \subset D_{n l}$. For each integer $n$ let $G_{n}^{\prime}$ be the sum of all the collections $G_{n l}^{\prime}$. It is evident that the sequence $\left\{G_{n}^{\prime}\right\}$ satisfies Conditions (1) and (2) of Axiom 1. To show that it satisfies Condition (3), let $R$ be an arbitrary line neighborhood, and let $x$ and $y$ be lines of $R$, distinct or not. It is sufficient to consider the case $x \neq y$. By Lemma 5 , there exists an open point set $D$ containing $x^{*}$ but not $y^{*}$. $D$ may obviously be assumed to lie within $R^{*}$. By Lemma 4 , there exists a line neighborhood $R^{\prime}$ containing $x$ such that $R^{* *} \subset D$.

Suppose there exists no integer $m$ such that, if $g$ is a line neighborhood of $G_{m}{ }^{\prime}$ containing $x$, then $\bar{g}$ is necessarily a subset of $R^{\prime}$. In this event there exists a sequence $\left\{g_{n}\right\}$ of line neighborhoods, where, for each $n, g_{n}$ belongs to $G_{n}{ }^{\prime}$, contains $x$, and $\bar{g}_{n}$ is not contained in $R^{\prime}$. Let $c_{n}$ be a line such that $g_{n}{ }^{*} \subset D_{n c_{n}}$. Since $x$ is in $g_{n}, x^{*} \subset D_{n c_{n}}$. Let $P$ and $Q$ be distinct points of $x$. Because $D_{n c_{n}}$ is a proper covering of $c_{n}$, there exist neighborhoods $U_{n}$ and $V_{n}$ of $G_{n}$ containing $P$ and $Q$ respectively and also containing points $P_{n}$ and $Q_{n}$ respectively of $c_{n}$. It follows by (3) of Axiom 1 that $\lim P_{n}=P$ and $\lim Q_{n}=Q$. Hence, by an argument similar to that used in the proof of Lemma 4 , if $E$ is an open set containing $x^{*}$, there exists an integer $m$ such that $c_{n}{ }^{*} \subset E$ for all $n \geqq m$.

Since $x$ is compact (Lemma 2), there exists $\dagger$ an open set $E$ containing $x$ such that $\bar{E} \subset R^{\prime *}$. Let $m_{0}$ be such an integer that, for all $n \geqq m_{0}, c_{n}^{*} \subset E$. The point set $x^{*}+c_{m_{0}}^{*}+c_{m_{0}+1}^{*}+\cdots$ is closed, compact, and contained in $E$. By Condition (4) of Axiom 1, there exists an integer $m \geqq m_{0}$ such that $D_{m x}+D_{m c_{0} 0}+D_{m c_{m_{0}+1}}+\cdots$ is contained in $E$. By Lemma $7,\left(\bar{g}_{m}\right)^{*} \subset \bar{E}$. Thus the original supposition is inadmissible and the proof is complete.

$\dagger$ M, Chapter I, Theorem 23. 
THEOREM 1a. There exists a sequence $\left\{G_{n}{ }^{\prime \prime}\right\}$ of collections of plane neighborhoods satisfying Conditions (1), (2), and (3) of Axiom 1 when $S$ is interpreted to be $G^{(2)}$ and "point" is interpreted to be "plane."

The proof is similar to the proof of Theorem 1.

Theorems 1 and 1a enable $\nmid$ us to quote theorems from Chapter I of $\mathbf{M}$ with regard to collections of lines in the line space $G^{(1)}$ and with regard to collections of planes in the plane space $G^{(2)}$.

Definition 6. Let $K$ be a point set and let $P$ be a limit point of $K$. A line $l$ is called a tangent line of $K$ at $P$ if, for an arbitrary point neighborhood $V$ of $P$ and an arbitrary line neighborhood $R$ of $l$, there exists a line of $R$ containing at least two distinct points of $K V$. The set of all tangent lines of $K$ at $P$ is called the paratingent of $K$ at $P$.

Defrnition 6a. Let $K$ be a point set and let $P$ be a limit point of $K$. A plane $p$ is called a tangent plane of $K$ at $P$ if, for an arbitrary point neighborhood $V$ of $P$ and an arbitrary plane neighborhood $R$ of $p$, there exists a plane of $R$ containing at least three non-collinear points of $K V$. The set of all tangent planes of $K$ at $P$ is called the biparatingent of $K$ at $P$.

Remark 5. For a line $l$ to be a tangent line of a point set $K$ at a limit point $P$ of $K$ it is necessary that $l$ contain $P$. It is evident that $K$ has no tangent line at a point which is not a limit point of $K$. Similar comments apply to tangent planes.

Theorem 2. The paratingent $\Lambda$ of a point set $K$ at a limit point $P$ of $K$ is closed.

If $l$ is a limit line of $\Lambda$, and if $R$ is a line neighborhood of $l$, then $R$ contains a line $l_{1}$ of $\Lambda$. Since $l_{1}$ is in $\Lambda$, there exists, for an arbitrary point neighborhood $V$ of $P$, a line $l^{\prime}$ of $R$ which contains two distinct points of $K V$. Thus $l$ is in $\Lambda$.

Theorem 2a. The biparatingent $\Pi$ of a point set $K$ at a limit point $P$ of $K$ is closed.

The proof is similar to the proof of Theorem 2.

REMARK 6. Definition 6 is not essentially altered if the clause "there exists a line of $R$ containing at least two distinct points of $K V$ " is replaced by the clause "there exists a line of $R$ which contains at least two distinct points of $K V$ or is a limit line of lines containing at least two distinct points of $K V$." This remark may be substantiated by an argument similar to that used in the proof of Theorem 2. A similar remark applies to Definition 6a. 
LEMMA 8. If $H$ is a collection of lines containing a common point $P$, then $a$ necessary condition that $H$ be the sum of two mutually separated $\dagger$ collections $M$ and $N$ is that $P$ be a cut point of the connected point set $H^{*}=M^{*}+N^{*}$.

Suppose that $H^{*}-P$ is connected. Then at least one of the sets $M^{*}-P$ and $N^{*}-P$, say the former, contains a limit point $Q$ of the other. It is evident that $P$ and $Q$ are $N$-related. By Lemma $6, \overline{P Q}$ is a limit line of $N$. But $\overline{P Q}$ is a line of $M$. This contradicts the hypothesis that $M$ and $N$ are mutually separated. Hence $H^{*}-P$ is not connected and $P$ is a cut point of the point set $H^{*}$.

Lемма 8a. If $H$ is a collection of planes containing a common line $l$, then a necessary condition that $H$ be the sum of two mutually separated collections $M$ and $N$ is that $l$ be a cut line of the connected line set $H^{* *}=M^{* *}+N^{* *}$, where $B^{* *}$ denotes the collection of all lines contained in any plane of $H$.

It follows from Lemma 9 below and the fact that a line is a continuum that if $A$ is a point and if $l$ is a line not containing $A$, the set of all lines containing $A$ and a point of $l$ is connected. Hence it follows that a plane is a connected set of lines. (Let $p$ be a plane and let $l$ and $l^{\prime}$ be distinct lines of $p$. Let $A$ and $B$ be points of $l$ and let $A^{\prime}$ and $B^{\prime}$ be points of $l^{\prime}$, where all four of these points are distinct. The set of all lines containing $A$ and a point of $\overline{A^{\prime} B}$ is connected; this set contains $l$ and $\overline{A A^{\prime}}$. The set of all lines containing $A^{\prime}$ and a point of $\overline{A B^{\prime}}$ is connected; this set contains $l^{\prime}$ and $\overline{A A^{\prime}}$. Hence the sum of these two sets is connected and contains $l$ and $l^{\prime}$.) It therefore follows that $H^{* *}$ is connected.

Now suppose that $H^{* *}-l$ is connected. Then at least one of the sets $M^{* *}-l$ and $N^{* *}-l$, say the former, contains a limit line $l^{\prime}$ of the other. The line $l^{\prime}$ is coplanar with $l$ and determines a plane $\overline{l l^{\prime}}$. If $P$ is a point of $l^{\prime}$ not in $l$, then, by Definition 2, $P$ is a limit point of the point set $N^{*}-l$. If $Q$ and $R$ are distinct points of $l$ not in $l^{\prime}$, then $P, Q$, and $R$ are $N$-related. The remainder of the proof follows the line of argument in the preceding proof.

Aхіом 2. The space $S$ is compact.

Theorem 3. The line space $G^{(1)}$ is compact.

Let $\left\{l_{n}\right\}$ be a sequence of distinct lines and let $\left\{P_{n}\right\}$ be a sequence of points with $P_{n}$ in $l_{n}$ for each $n$. By Axiom 2, some subsequence $\left\{P_{n_{i}}\right\}$ of $\left\{P_{n}\right\}$ has a sequential limit $P$. By Remark 4, there exists a neighborhood $V$ of $P$ which contains no line. Let $\left\{Q_{n_{i}}\right\}$ be a sequence of points where, for each $i$, $Q_{n_{i}}$ is in $l_{n_{i}}$ and not in $V$. By Axiom 2, some subsequence of $\left\{Q_{n_{i}}\right\}$ has a

$\dagger$ Two sets are said to be mutually separated if they are mutually exclusive and if neither contains a limit element of the other. 
sequential limit $Q$, and $Q \neq P$. By Lemma 6 , some subsequence of $\left\{l_{n}\right\}$ has a sequential limit. An obvious extension of this argument proves

ThEOREM 3a. The plane space $G^{(2)}$ is compact.

Notation. If $K$ is a point set and if $A$ is a point, then $K ; A$ denotes the closure of the set of all lines containing $A$ and a point of $K-K A$. If $B$ and $B^{\prime}$ are points of $K$ distinct from $A$, and if there exists a connected subset of $K ; A$ containing $\overline{B A}$ and $\overline{A B^{\prime}}$, then $K ; B, A, B^{\prime}$ denotes the component of $K ; A$ containing $\overline{B A}$ and $\overline{A B^{\prime}}$. If $k$ is a set of lines with a common point $A$ and if $a$ is a line containing $A$, then $k ; a$ denotes the closure of the set of all planes containing $a$ and a line of $k-k a$. If $b$ and $b^{\prime}$ are lines of $k$ distinct from $a$, and if there exists a connected subset of $k ; a$ containing $\overline{b a}$ and $\overline{a b^{\prime}}$ (where $\overline{b a}$ is the plane containing $b$ and $a$ ), then $k ; b, a, b^{\prime}$ denotes the component of $k ; a$ containing $\overline{b a}$ and $\overline{a b^{\prime}}$.

LemMa 9. If $K$ is a connected point set, if $A$ is a point, and if $B$ and $B^{\prime}$ are points of $K$ distinct from $A$, then either $K ; B, A, B^{\prime}$ exists or else $K ; A, B, B^{\prime}$ and $K ; B, B^{\prime}, A$ exist.

It may be assumed that $A, B$, and $B^{\prime}$ are non-collinear, as otherwise the lemma is trivial. Suppose there exists no connected subset of $K ; A$ containing $\overline{B A}$ and $\overline{A B^{\prime}}$. Since $K ; A$ is closed and compact, $K ; A$ consists $\dagger$ of the sum of two mutually separated line sets $M$ and $N$ containing $\overline{B A}$ and $\overline{A B^{\prime}}$ respectively. By Lemma 8 it follows that $M^{*}-A$ and $N^{*}-A$, and hence $M^{*}(K-K A)$ and $N^{*}(K-K A)$, are mutually separated. Hence $A$ is a point of $K$ and $A$ separates $B$ and $B^{\prime}$ in $K$. By a well known theorem, $\ddagger B$ does not separate $A$ and $B^{\prime}$ in $K$, and $B^{\prime}$ does not separate $A$ and $B$ in $K$. Therefore $K ; A, B, B^{\prime}$ and $K ; B, B^{\prime}, A$ exist.

LEMMA 9a. If $K$ is a connected set of lines with a common point $A$, if $a$ is a line containing $A$, and if $b$ and $b^{\prime}$ are lines of $k$ distinct from $a$, then either $k ; b, a, b^{\prime}$ exists or else $k ; a, b, b^{\prime}$ and $k ; b, b^{\prime}, a$ exist.

The proof is exactly parallel to the proof of Lemma 9.

Notation. If $K$ is a connected point set, if $A$ is a point, and if $B$ and $B^{\prime}$ are points of $K$ distinct from $A$, then $K ; B-A-B^{\prime}$ denotes $K ; B, A, B^{\prime}$ if this set exists and $K ; A, B, B^{\prime}+K ; B, B^{\prime}, A$ otherwise. Since $K ; A, B, B^{\prime}$ and $K ; B, B^{\prime}, A$ have a common line, it follows by Lemma 9 that $K ; B-A-B^{\prime}$ always exists, contains $\overline{B A}$ and $\overline{A B^{\prime}}$, is connected, and each line of $K ; B-A-B^{\prime}$ contains at least two distinct points of the point set $K+A$ or is a limit line of such lines.

$\dagger$ M, Chapter I, Theorem 35.

$\ddagger$ M, Chapter I, Theorem 49. 
LEMмa 10. If $\left\{M_{n}\right\}$ is a sequence of connected sets of lines (planes), and if there exists a convergent sequence $\left\{m_{n}\right\}$ of lines (planes) with $m_{n}$ in $M_{n}$ for each $n$, then the limiting set of $\left\{M_{n}\right\}$ is a line (plane) continuum.

This lemma occurs in M, Chapter I, as Theorem 42.

TheOREM 4. If $K$ is either a locally connected point set or a point continuum, and if $P$ is a limit point of $K$, then the paratingent $\Lambda$ of $K$ at $P$ is a continuum of lines.*

The proof of this theorem is carried out first for the case where $K$ is a continuum. Let $\left\{V_{n}\right\}$ be a sequence of point neighborhoods which closes down on $P$. Let $\rho$ and $\sigma$ be distinct lines of $\Lambda$ (assuming $\Lambda$ to contain more than one line). Let $\left\{R_{n}\right\}$ and $\left\{S_{n}\right\}$ be sequences of line neighborhoods closing down on $\rho$ and $\sigma$ respectively. By Definition 6, there exists a pair of distinct points $A_{n}$ and $B_{n}$ in $K V_{n}$ such that the line $\overline{A_{n} B_{n}}$ is in $R_{n}$; likewise there exists a pair of distinct points $C_{n}$ and $D_{n}$ in $K V_{n}$ such that the line $\overline{C_{n} D_{n}}$ is in $S_{n}$. Of the two points $C_{n}$ and $D_{n}$, let $C_{n}$ be taken as one which is distinct from $B_{n}$. Let it be supposed that $V_{1}$ was so chosen that $K$ contains a point outside $V_{1}$. For $n>1$, let $\beta_{n}\left(\gamma_{n}\right)$ be that component of $K \bar{V}_{2}$ containing $B_{n}\left(C_{n}\right)$. Since $K$ is a continuum, there exists $\nmid$ a point $B_{n}^{\prime}\left(C_{n}^{\prime}\right)$ of $\beta_{n}\left(\gamma_{n}\right)$ on the boundary of $V_{2}$. Since the boundary of $V_{2}$ is compact, there exists a subsequence $\left\{n_{i}\right\}$ of the sequence $\{n\}$ such that the sequence $\left\{B_{n_{i}^{\prime}}\right\}$ has a sequential limit $B^{\prime}$; likewise, there exists a subsequence $\left\{n_{i_{j}}\right\}$ of $\left\{n_{i}\right\}$ such that the sequence $\left\{C_{\alpha}\right\}$ has a sequential limit $C^{\prime}$, where $\alpha$ runs (both now and in what follows) through the sequence $\left\{n_{i_{j}}\right\}$. By Lemma 9 , there exist, for each $\alpha$, connected line sets $\beta_{\alpha} ; B_{\alpha}-A_{\alpha}-B_{\alpha}^{\prime}, \beta_{\alpha} ; B_{\alpha}-C_{\alpha}-B_{\alpha}^{\prime}, \gamma_{\alpha} ; C_{\alpha}-B_{\alpha}-C_{\alpha}^{\prime}$, and $\gamma_{\alpha} ; C_{\alpha}-D_{\alpha}-C_{\alpha}^{\prime}$, where each line of each of these four sets contains two distinct points of $K V_{1}$ or is a limit line of such lines. Since $\beta_{\alpha} ; B_{\alpha}-C_{\alpha}-B_{\alpha}^{\prime}$ and $\gamma_{\alpha} ; C_{\alpha}-B_{\alpha}-C_{\alpha}^{\prime}$ have the line $\overline{B_{\alpha} C_{\alpha}}$ in common, $\beta_{\alpha} ; B_{\alpha}-C_{\alpha}-B_{\alpha}^{\prime}+$ $\gamma_{\alpha} ; C_{\alpha}-B_{\alpha}-C_{\alpha}^{\prime}$ is connected. But $\lim \overline{B_{\alpha} A_{\alpha}}=\rho$ and $\lim \overline{A_{\alpha} B_{\alpha}^{\prime}}=\overline{P B^{\prime}}$. Hence $\rho$ and $\overline{P B^{\prime}}$ are lines of the limiting set $\Sigma^{1}$ of the sequence $\left\{\beta_{\alpha} ; B_{\alpha}-A_{\alpha}-B_{\alpha}^{\prime}\right\}$. Similarly, $\overline{P B^{\prime}}$ and $\overline{P C^{\prime}}$ are lines of the limiting set $\Sigma^{2}$ of $\left\{\beta_{\alpha} ; B_{\alpha}-C_{\alpha}-B_{\alpha}^{\prime}+\right.$ $\left.\gamma_{\alpha} ; C_{\alpha}-B_{\alpha}-C_{\alpha}^{\prime}\right\}$, and $\overline{P C^{\prime}}$ and $\sigma$ are lines of the limiting set $\Sigma^{3}$ of $\left\{\gamma_{\alpha} ; C_{\alpha}-D_{\alpha}-C_{\alpha}^{\prime}\right\}$. By Lemma $10, \Sigma^{1}, \Sigma^{2}$, and $\Sigma^{3}$ are line continua; because pairs of these sets have common lines, their sum $\lambda_{1}=\Sigma^{1}+\Sigma^{2}+\Sigma^{3}$ is a line continuum containing $\rho$ and $\sigma$. Every line of $\lambda_{1}$ contains at least two distinct points of $K V_{1}$ or is a limit of such lines. Let $\lambda_{n}$ be the line continuum obtained from $K V_{n}$ by the same process that led to $\lambda_{1}$ from $K V_{1}$. By Lemma 10, the

* The central idea underlying the proof of this theorem was suggested by a proof of Mirguet, Annales de l'École Normale Supérieure, vol. 51 (1934), p. 201.

$\dagger \mathrm{M}$, Chapter I, Theorem 40. 
limiting set $\lambda$ of the sequence $\left\{\lambda_{n}\right\}$ is a line continuum; it is evident that $\lambda$ contains $\rho$ and $\sigma$. But by Remark 6, each line of $\lambda$ is a tangent line of $K$ at $P$. Hence $\Lambda$ is connected. By Theorem 2, $\Lambda$ is closed. Therefore $\Lambda$ is a continuum of lines.

The proof of the theorem in the case where $K$ is locally connected is similar to the above proof. Within each neighborhood $V_{n}$ of the above sequence $\left\{V_{n}\right\}$ there exists a neighborhood $V_{n}^{\prime}$ of $P$ such that $K V_{n}^{\prime}$ is connected. The points $A_{n}, B_{n}, C_{n}$, and $D_{n}$ may be taken in $K V_{n}^{\prime}$. By Lemma 9 , the sets $K V_{n}^{\prime} ; A_{n}-B_{n}-C_{n}$ and $K V_{n}^{\prime} ; B_{n}-C_{n}-D_{n}$ exist. Since these sets have the common line $\overline{B_{n} C_{n}}$, their sum is connected. The limit process based on Lemma 10 may be applied as before to these sets.

Notation. If $K$ is a point set, if $A$ and $B$ are distinct points, and if $C$ and $C^{\prime}$ are points of $K$ not in $\overline{A B}$, then $(K ; A) ; \overline{A C}, \overline{A B}, \overline{A C^{\prime}}$, if it exists, will be denoted by

$$
K ; \underset{B}{C, A, C^{\prime}} \text {. }
$$

If $K$ is connected then, by Lemma.9, either

$$
K ; C, A, C^{\prime} \text { exists, or }
$$

$$
K ; A, C, C^{\prime}+K ; C, C^{\prime}, A \text { exists. }
$$

In Case (1) it follows by Lemma 9a that either

$$
\begin{aligned}
& K ; \underset{B}{C, A, C^{\prime}} \text { exists, or } \\
& K ; \underset{C}{B, A, C^{\prime}}+K ; \underset{C^{\prime}}{C, A} \text { exists. }
\end{aligned}
$$

In Case (2) either

$$
\begin{aligned}
& K ; \underset{B}{A, C, C^{\prime}}+K \underset{B}{C, C^{\prime}, A} \text { exists, or } \\
& K \underset{A}{B, C, C^{\prime}}+K ; \underset{C^{\prime}}{A, C}+K ; \underset{B}{C, C^{\prime}, A} \text { exists, or } \\
& K ; \underset{B}{A, C, C^{\prime}}+K ;{ }_{C}^{B, C^{\prime}, A}+K ;{ }_{A}^{C, C^{\prime}, B} \text { exists, or } \\
& K ; \underset{A}{B, C, C^{\prime}}+K ;{ }_{C^{\prime}}^{A, C, B}+K ; \underset{C}{B, C^{\prime}, A}+K{ }^{C} \underset{A}{C, C^{\prime}, B} \text { exists. }
\end{aligned}
$$

Of the six sets (1a) to (2d), let 


$$
K ; C-{ }_{B}^{A}-C^{\prime}
$$

denote the first one existing. If $C$ and $C^{\prime}$ are collinear with $A$ or $B$, then

$$
K ; C-\stackrel{A}{B}-C^{\prime}
$$

denotes the single plane $\overline{A B C}=\overline{A B C^{\prime}}$. Thus, for any connected point set $K$ and for points $A, B, C$, and $C^{\prime}$ as described above,

$$
K ; C-\stackrel{A}{B}-C^{\prime}
$$

always exists, contains $\overline{C A B}$ and $\overline{A B C^{\prime}}$, is connected, and each plane of

$$
K ; C-{ }_{B}^{A}-C^{\prime}
$$

contains at least three non-collinear points of $K+A+B$ or is a limit plane of such planes.

The extension of Theorem 4 to tangent planes in the case where $K$ is a point continuum seems to present difficulty in that the non-collinear point triples needed for the proof may not exist. However, the following lemma makes it possible to prove the extension in two special cases of interest.

LEMMA 11. If $P$ is a point, if $\left\{\alpha_{n}\right\}$ and $\left\{\beta_{n}\right\}$ are two sequences of point sets, if the sequential limiting set of $\left\{\alpha_{n}\right\}$ contains a point $A$ distinct from $P$, if the limiting set of every subsequence of $\left\{\beta_{n}\right\}$ contains a point distinct from $P$, and if it is impossible to find a subsequence $\left\{n_{i}\right\}$ of $\{n\}$ and sequences $\left\{A_{n_{i}}\right\}$ and $\left\{B_{n_{i}}\right\}$ such that $\lim \overline{A_{n_{i}} B_{n_{i}}}$ exists and does not contain $P$, where, for each $n_{i}, A_{n_{i}}$ is a point of $\alpha_{n_{i}}, B_{n_{i}}$ is a point of $\beta_{n_{i}}$, and $A_{n_{i}} \neq B_{n_{i}}$, then the limiting sets of $\left\{\alpha_{n}\right\}$ and $\left\{\beta_{n}\right\}$ are contained in the line $\overline{P A}$.

The proof of this lemma is readily effected by first showing that an arbitrary point of the limiting set of $\left\{\beta_{n}\right\}$ is contained in $\overline{P A}$ and then using this result to show that an arbitrary point of the limiting set of $\left\{\alpha_{n}\right\}$ is contained in $\overline{P A}$.

Let $K$ be a point continuum and let $P$ be a point of $K$. Let $\left\{V_{n}\right\}$ be a sequence of point neighborhoods closing down on $P$. Let $\rho$ and $\sigma$ be distinct planes of the biparatingent $\Pi$ of $K$ at $P$. Let $\left\{R_{n}\right\}$ and $\left\{S_{n}\right\}$ be sequences of plane neighborhoods closing down on $\rho$ and $\sigma$ respectively. By Definition 6a, there exist three non-collinear points $A_{n}, B_{n}$, and $C_{n}$ in $K V_{n}$ such that the plane $\overline{A_{n} B_{n} C_{n}}$ is in $R_{n}$; likewise there exist three non-collinear points $X_{n}, Y_{n}$, and $Z_{n}$ in $K V_{n}$ such that the plane $\overline{X_{n} Y_{n} Z_{n}}$ is in $S_{n}$. Let $\alpha_{n}, \beta_{n}, \gamma_{n}, \xi_{n}, \eta_{n}, \zeta_{n}$ be those components of $K \bar{V}_{2}$ containing $A_{n}, B_{n}, C_{n}, X_{n}, Y_{n}$, and $Z_{n}$. 
If no non-degenerate subcontinuum of that part of $K$ within some neighborhood $V$ of $P$ is contained in a line, and if $V_{1}$ is taken within $V$, then by Lemma 11 it is possible to find a subsequence $\left\{n_{i}\right\}$ of $\{n\}$ and points $B_{n_{i}}^{\prime}$, $C_{n_{i}^{\prime}}, X_{n_{i}^{\prime}}$, and $Y_{n_{i}^{\prime}}$ of $\beta_{n_{i}}, \gamma_{n_{i}}, \xi_{n_{i}}$, and $\eta_{n_{i}}$ such that $\lim \overline{B_{n_{i}}^{\prime} C_{n_{i}^{\prime}}^{\prime}}, \lim \overline{C_{n_{i}}^{\prime} X_{n_{i}^{\prime}}^{\prime}}$, and $\lim \overline{X_{n i}^{\prime} Y_{n_{i}^{\prime}}}$ do not contain $P$. Hence there exists a set of connected sets of planes of the following sort for sufficiently large $n_{i}$ :

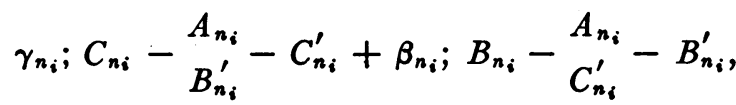

$$
\begin{aligned}
& \beta_{n_{i}} ; B_{n_{i}}^{\prime}-\begin{array}{l}
X_{n_{i}} \\
C_{n_{i}}^{\prime}
\end{array}-B_{n_{i}}+\xi_{n_{i}} ; X_{n_{i}}-\begin{array}{l}
B_{n_{i}} \\
C_{n_{i}}^{\prime}-X_{n_{i}}^{\prime},
\end{array}
\end{aligned}
$$

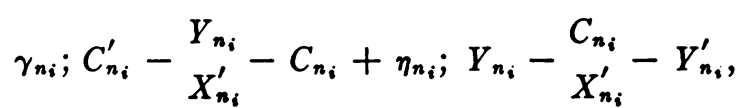

$$
\begin{aligned}
& \eta_{n_{i}} ; Y_{n_{i}}^{\prime}-\frac{X_{n_{i}}^{\prime}}{Z_{n_{i}}}-Y_{n_{i}}+\xi_{n_{i}} ; X_{n_{i}}^{\prime}-\frac{Y_{n_{i}}}{Z_{n_{i}}}-X_{n_{i}} .
\end{aligned}
$$

If the preceding hypothesis concerning the subcontinua of $K$ is not valid, it is still possible to obtain a set of planes of the form (1) by imposing conditions on the lines and planes of the space $S$ like the following: (a) if $P$ is a point of the space $S$, there exists a point neighborhood $V$ of $P$ such that, if $\pi$ is a plane containing a point of $V$, then $V-V \pi$ is the sum of two mutually separated point sets; and (b) a line is a simple continuous arc. Having obtained the set (1), one may prove the extension of Theorem 4 by following the line of argument in the proof of Theorem 4.

Theorem 4a. If $K$ is a locally connected point set and if $P$ is a limit point of $K$, then the biparatingent $\Pi$ of $K$ at $P$ is a continuum of planes.

The proof of this theorem is effected by taking neighborhoods $V_{n}^{\prime}$ of $P$ within $V_{n}$ such that $K V_{n}^{\prime}$ is connected and by modifying the above argument in exactly the same way as in the last paragraph of the proof of Theorem 4.

It is readily possible to define hyperplanes of every finite "dimension" with the aid of Definition 2 and the inductive process indicated in Definition 4a. Most of the results of this paper may be extended by means of these hyperplanes.

Case School of Applied Science,

Cleveland, OHo 